\title{
Continuous EEG Source Imaging enhances analysis of EEG-fMRI in focal epilepsy
}

${ }^{1,2}$ S. Vulliemoz, ${ }^{1}$ R. Rodionov, ${ }^{1} \mathrm{DW}$. Carmichael, ${ }^{1} \mathrm{R}$. Thornton, ${ }^{4} \mathrm{M}$. Guye, ${ }^{5} \mathrm{SD}$. Lhatoo, ${ }^{3} \mathrm{CM}$. Michel, ${ }^{1}$ JS. Duncan, ${ }^{1}$ L. Lemieux

${ }^{1}$ National Society for Epilepsy MRI Unit, Department of Clinical and Experimental Epilepsy UCL Institute of Neurology and National Hospital for Neurology and Neurosurgery, Queen Square, London, WC1N 3BG, United Kingdom

${ }^{2}$ Presurgical Evaluation for Epilepsy Unit and ${ }^{3}$ Functional Brain Mapping Laboratory, Neurology Department, University Hospital and University of Geneva, Switzerland

${ }^{4}$ INSERM U 751 - Service de Neurophysiologie Clinique \& CNRS UMR 6612, Faculté de Médecine \& CHU Timone, Marseille, France

${ }^{5}$ Department of Neurology, North Bristol NHS Trust, Frenchay Hospital, Frenchay Road, Bristol, United Kingdom

This work was undertaken at the National Society for Epilepsy MRI Unit, Department of Clinical and Experimental Epilepsy UCL Institute of Neurology, United Kingdom

Running title: EEG Source Imaging for EEG-fMRI analysis

Key words: EEG, source imaging, epilepsy, interictal, fMRI,

Abstract: 248 words

Main text: 4685 words

Tables: 3; Figures: 6

References: 45

Corresponding author:

Louis Lemieux

MRI Unit, National Society for Epilepsy

Chesham Lane

Chalfont St Peter

Buckinghamshire SL9 ORJ

United Kingdom

I.lemieux@ion.ucl.ac.uk

Tel: ++44 1494601300

Fax: ++441494875666 


\section{ABSTRACT}

Introduction: EEG-correlated fMRI (EEG-fMRI) studies can reveal haemodynamic changes associated with Interictal Epileptic Discharges (IED). Methodological improvements are needed to increase sensitivity and specificity for localising the epileptogenic zone. We investigated whether the estimated EEG source activity improved models of the BOLD changes in EEG-fMRI data, compared to conventional «event-related » designs based solely on the visual identification of IED.

Methods: Ten patients with pharmaco-resistant focal epilepsy underwent EEG-fMRI. EEG Source Imaging (ESI) was performed on intra-fMRI averaged IED to identify the irritative zone. The continuous activity of this estimated IED source (cESI) over the entire recording was used for fMRI analysis (cESI model). The maps of BOLD signal changes explained by cESI were compared to results of the conventional IED-related model.

Results: ESI was concordant with non-invasive data in 13/15 different types of IED. The cESI model explained significant additional BOLD variance in regions concordant with videoEEG, structural MRI or, when available, intracranial EEG in 10/15 IED. The cESI model allowed better detection of the BOLD cluster, concordant with intracranial EEG in 4/7 IED, compared to the IED model. In 4 IED types, cESI-related BOLD signal changes were diffuse with a pattern suggestive of contamination of the source signal by artefacts, notably incompletely corrected motion and pulse artefact. In one IED type, there was no significant BOLD change with either model.

Conclusion: Continuous EEG source imaging can improve the modelling of BOLD changes related to interictal epileptic activity and this may enhance the localisation of the irritative zone. 


\section{ACKNOWLEDGEMENTS}

We would like to thank Prof. Karl Friston, Wellcome Trust Centre for Neuroimaging, London, for his precious methodological advice during the SPM methods meeting. We would also like to thank Prof. Matthew Walker, Dr Sheilagh Smith, Dr Beate Diehl, and Catherine Scott for clinical and electrophysiological discussions. We thank Mr Andrew McEvoy, Dr Jean Régis and Mr David Sandeman for intracranial electrode implantations in London, Marseille and Bristol. The Cartool software has been programmed by D. Brunet, from the Functional Brain Mapping Laboratory, Geneva, Switzerland, and is supported by the Center for Biomedical Imaging (CIBM) of Geneva and Lausanne. S. Vulliemoz is supported by the "Fonds de Perfectionnement" of the University Hospital of Geneva and by a fellowship for advanced researcher from the Swiss National Science Foundation. We acknowledge the financial support of the Swiss National Science Foundation No. 32-111783, 33CM20-124115, UK Medical Research Council (MRC grant G0301067), the Center for Biomedical Imaging (CIBM) of Geneva and Lausanne. We are grateful to the Big Lottery Fund, Wolfson Trust and National Society for Epilepsy for supporting the 3T MRI scanner at NSE. This work was undertaken at UCLH/UCL who received a proportion of funding from the Department of Health's NIHR Biomedical Research Centres funding scheme. None of the authors has any conflict of interest to disclose. 


\section{INTRODUCTION}

Around $30 \%$ of patients with epilepsy suffer from recurring seizures despite treatment with multiple anti-epileptic drugs (Sillanpaa 2000). Some of these patients can benefit from epilepsy surgery if the epileptogenic focus can be precisely localised. EEG-correlated fMRI (EEG-fMRI) is a non-invasive imaging technique that may indicate regions of increased or decreased cerebral oxygenation (Blood Oxygen Level Dependant, BOLD, signal) correlated with Interictal Epileptiform Discharges (IED) (Al-Asmi, et al. 2003; Salek-Haddadi, et al. 2006). At present, EEG-fMRI has limited sensitivity, with $40-70 \%$ of EEG-fMRI datasets containing focal IED showing significant BOLD changes. The regions of significant BOLD change have been shown to be generally concordant with the findings of conventional electro-clinical and imaging data, and in particular with intracranial electrodes recording of IED (Benar, et al. 2006).

Most interictal EEG-fMRI studies are based on event-related fMRI designs with identification of IED by expert observers and the representation of the IED as idealised (and usually uniform) 'stick functions', convolved with a model of the haemodynamic response function (Al-Asmi, et al. 2003). For prolonged runs of IED, a block design that takes into account the duration of the discharges has been shown increase sensitivity of EEG-fMRI analysis (Bagshaw, et al. 2005; Salek-Haddadi, et al. 2006). These models, however, do not account for variations of the BOLD response to individual IED, although amplitude and morphology of IED on scalp EEG have been reported to be different in patients with and without BOLD response to IED (Krakow, et al. 1999). Moreover, modelling only IED detected on scalp EEG would often not reflect the abundant underlying epileptic activity that can be recorded by intracranial EEG (Tao, et al. 2005).

In the present study, we used continuous Electrical Source Imaging (cESI) to obtain a continuous estimate of the activity of the IED source. We then used this activity function as a parametric model of the BOLD signal changes to address the following questions:

1) Does the proposed parametric modelling of epileptic activity explain additional variance of the BOLD compared to a modelling of IED as discrete events? 
2) Is there spatial concordance between the ESI-correlated BOLD signal changes, the source of IED onset defined by ESI and other non-invasive or invasive imaging modalities? In other words, does cESI reflect ongoing epileptic activity? 


\section{METHODS}

\section{Patients and electro-clinical data}

Patients with refractory focal epilepsy undergoing pre-surgical assessment were selected from the pool of EEG-fMRI data acquired on a 3T MR scanner between January 2004 and October 2008 according to the following criteria: 1) intra-MRI EEG recording with 32 electrodes or more; 2) presence of spikes, spike-waves or sharp waves on the intra-MRI EEG. Epileptic transients in the form of short runs of low amplitude high frequency polyspikes were not considered as they have poor signal-to-noise ratio for ESI.

In 10 patients fulfilled the criteria (one EEG-fMRI recording each), we identified 15 separate IED types that were used for analysis, resulting in 15 IED-type specific analysis. Clinical, electrophysiological and imaging data of the patients are given in Table 1. All patients had cryptogenic focal epilepsy except for patient 4 who had focal cortical dysplasia in the left occipital cortex, patient 7 who had left hippocampal sclerosis and patient 10 who had a left frontal sub-cortical lesion (detectable as increased signal on FLAIR images). The study was approved by the Research Ethics Committee of the UCL Institute of Neurology and UCL Hospitals. Written informed consent was obtained from all patients.

\section{EEG-fMRI set-up and acquisition}

EEG in resting state with closed eyes outside (15 minutes prior to scanning) and inside the scanner was acquired using MR-compatible EEG system (Brain Products, Munich, Germany). A 32- or 64-electrode EEG cap was used according to the 10-20 or 10-10 electrode position convention. All patients underwent EEG-fMRI on a 3T Signa Excite HDX scanner (GE Medical Systems, Milwaukee) and were asked to lie still in the scanner with their eyes closed and no specific instruction regarding vigilance. EEG was recorded continuously during fMRI. ECG was recorded with a single lead. Each patient underwent 2 or 3 20-minute blocks of EEG-fMRI acquisition. Each fMRI dataset consisted of $404 \mathrm{~T}^{*}$ weighted single-shot gradient-echo echo-planar images (EPI; TE/TR 30/3000 ms, flip angle 90 , FOV $24 \times 24 \mathrm{~cm}^{2}, 43$ or 44 interleaved slices with in-plane resolution $3 \times 3 \mathrm{~mm}^{2}$ and $3 \mathrm{~mm}$ thickness). For the purpose of anatomical localisation and EEG source localisation a T1 
FSPGR image (TE/TR/TI=3.1/8.2/450 ms; $256 \times 256 \times 170$ matrix, resolution: $\left.1.1 \times 1.1 \times 1.1 \mathrm{~mm}^{3}\right)$ including nasion and inion (resampled offline to $1 \times 1 \times 1 \mathrm{~mm}^{3}$ voxels for comparison with isotropic images created with the head model for ESI).

\section{EEG analysis and Electrical Source Imaging (ESI)}

The EEG was corrected offline for gradient artefact and pulse-related artefacts using average artefact subtraction methods described elsewhere (Allen, et al. 2000; Allen, et al. 1998). IED were identified and marked by two experienced electro-encephalographers (SV, RT) (SalekHaddadi, et al. 2006).

The IED used for fMRI modelling were analysed using an ESI methodology implemented in Cartool (http://brainmapping.unige.ch/Cartool.htm). After filtering (high-pass: $0.3 \mathrm{~Hz}$, lowpass: $35 \mathrm{~Hz}$ ), IED were averaged and channels containing artefacts were interpolated. We used a Spherical head Model with Anatomical Constraints (SMAC) based on a 3-shell spherical realistic head model and the patient's individual MRI (Spinelli, et al. 2000). The source space was limited to the grey matter, segmented using the Statistical Parametric Mapping software SPM5 (www.fil.ion.ucl.ac.uk/spm). A standard localisation of the electrodes according to the 10-20 system was assumed to obtain electrode coordinates. Estimation of the location of intracranial generators was carried out using a linear distributed inverse solution (LAURA) (Grave de Peralta Menendez, et al. 2001) This source imaging incorporates the physical law that the strength of a source regresses regularly with distance by a local autoregressive average with coefficients depending on the distance between solution points (Michel et al., 2004). The parameters used for the LAURA calculation were fixed to a neighbourhood size of 26 solution points and a regression with the inverse of the cubic distance (for vector fields). The regularization parameter was fixed to Alpha $=1$ for all maps (Pasqual Marqui, et al. 2009). To estimate the IED source, we used the time point corresponding to the half maximum of the first rising phase of the global field power of the averaged IED (Lantz, et al. 2003). 


\section{Source activity based on continuous ESI (cESI)}

The analysis strategy for EEG, ESI and fMRI analysis is summarised in Figure 1. For each IED type, we defined a region of interest (ROI) consisting of 5 neighbouring ESI solution points around the maximum estimated current density at ESI onset. Due to the local dependency constraint that is implemented in the LAURA source localisation algorithm, the selected ROI is representative of a larger area of activity. The averaged current density of this ROI was determined for each time point of the whole intra-MR EEG recording, giving the function cESI (Figure 1). In each case, a control ROI was identified as the region of minimal increase of activity during IED, and the source activity for this region was labelled $c E S I_{c}$. This $\mathrm{CESI}_{\mathrm{C}}$ parameter was intended to account for artefacts in the source activity signal which may arise from motion, myogenic artefacts or pulse artefacts (similarly to the EEG trace) thereby potentially affecting source estimation diffusely throughout the brain. The choice of a single control region might not represent the global artifact or fluctuation of physiological activity but this represents a compromise to ensure a good degree of specificity of the cESI regressor to epileptic activity, while avoiding a strong reduction of sensitivity due to the spatial dependency constraint of ESI mentioned above.

\section{fMRI analysis}

All fMRI analyses were performed using SPM5. All EPI images were first realigned to the mean image, re-sliced and smoothed with a cubic Gaussian kernel of $8 \mathrm{~mm}$ full width at half maximum.

\section{1) Parametric model: cESI model:}

An embedded modelling strategy was employed to assess the contribution of the source activity regressors to the mapping of network involved in interictal epileptic activity. A General Linear Model (GLM) was built, including the effects of the individual IED, each represented as a stick function, $\mathrm{cESI}$ and $\mathrm{cESI} \mathrm{c}_{\mathrm{c}}$, convolved with the canonical $\mathrm{HRF}$, its time derivative and dispersion derivative to account for variable slice delay and variations of the HRF across brain regions and individuals. Subsequently, the convolved $\mathrm{cESI}$ and $\mathrm{cESI}_{\mathrm{c}}$ functions were down-sampled to the repetition time of the $\mathrm{fMRI}$ sequence (TR) to yield parametric 
regressors. Convolution and down-sampling were performed in Matlab 7.3 (Mathworks, Natick, MA, USA). The IED and cESI regressors represent effects of interest, and $c E S I_{c}$ represents a confounding effect. We also modelled the following set of confounds as previously published by our group (Salek-Haddadi, et al. 2006) : 24 parameters representing a Volterra-expansion of the 6 rigid-body realignment parameters (Friston, et al. 1996), cardiac effects (Liston, et al. 2006b) and 'scan nulling' regressors (Heaviside function) to account for the effects of large motion events $(>=0.5 \mathrm{~mm}$ inter-scan displacement along any axis) (Friston, et al. 1996; Lemieux, et al. 2007). In addition, 'EEG nulling' regressors were used to model events in which the EEG was contaminated by artefacts. For this purpose, the EEG and cESI signal were reviewed in parallel and artefact-contaminated segments were visually identified. A Heaviside function (similarly to the 'scan nulling' regressors) which covered the duration of the scan corresponding to the artefact-affected event and the following two scans, was introduced in the GLM as a way of limiting the impact of these artefacts on the correlation between cESI and BOLD signals. In datasets containing more than one type of IED with different localisation (i.e. with different presumed underlying generators) one GLM was built for each type of IED. Baseline drift was removed by applying a high-pass filter with a cut-off of $1 / 128 \mathrm{~Hz}$.

For each cESI model we obtained two $\operatorname{SPM}\{F\}$ contrast maps to estimate the following effects: 1) cESI-related BOLD signal changes to show variance of the BOLD signal related to the activity of the estimated IED source (cESI SPM\{F\} maps); 2) combined IED and cESI (cESI+IED SPM $\{F\}$ maps) to map BOLD signal changes related to all available information about epileptic activity. The resulting maps were corrected for multiple voxel comparisons (Family wise error, $\mathrm{p}<0.05)$.

\section{2) Conventional IED model:}

This "conventional" model comprised only regressors for the IED (convolved with HRF, time and dispersion derivatives), 24 realignment parameters, cardiac effects, 'scan nulling' regressors and high-pass filter, each as described in the section on the parametric model. 
Results of ESI and IED model have already been published elsewhere (Vulliemoz, et al. 2009) for all patients except patient 7.

\section{Assessment of Concordance}

For each model, the three resulting $\operatorname{SPM}\{F\}$ maps were evaluated for spatial concordance with prior hypothesis regarding localisation of the epileptic focus. Since only $4 / 10$ patient had concordant intracranial EEG recording and only 3/10 had surgical resection we based our concordance assessment on non-invasive electro-clinical data and structural imaging with validation from intracranial EEG when available.

We labelled as "Concordant (C)" the maps in which all statistically significant clusters (excepting those belonging to the $\mathrm{DMN}$ ) were was located in the same lobe as prior knowledge of focus localisation.. The "Concordance plus (C+)" label was applied to maps in which the most statistically significant cluster was located in the same lobe as prior knowledge of focus localisation but where additional clusters were present in other lobes than the presumed focus but in regions known to be structurally and functionally connected to it (i.e. limbic network in temporal lobe epilepsy, contralateral frontal lobe in frontal lobe epilepsy, occipito-temporal distribution in occipital lobe epilepsy). Diffuse BOLD changes involving all lobes bilaterally were labelled as "Discordant (D)". In some cases, BOLD maps showed clusters clearly belonging to the Default Brain Network (DMN: medial parietal = precuneus, bilateral associative temporal-parietal cortex, bilateral medial fronto-polar, thalamus) (Raichle, et al. 2001). These were not considered for the assessment of concordance, even when they included the global statistical maximum. These regions exhibit haemodynamic changes that are time-locked to IED in TLE (Laufs, et al. 2006) or in bilateral spike-wave discharges (Hamandi, et al. 2006). Although their role in IED generation is unclear, these brain regions are involved in the modulation of brain activity that strongly interact with, but are not part of, the irritative zone.

The same criteria for concordance were used to label the ESI solutions with respect to the presumed or confirmed localisation of the epileptic focus. 
To assess the potential clinical relevance of the cESI model, we also determined if the concordant BOLD clusters obtained with the cESI model gave additional information regarding the localisation of the IED generators, as compared with the conventional IED model. We restricted this comparison to cases in which intracranial EEG was available as a gold standard localising tool.

\section{Exploration of factors that influence the degree of concordance:}

We considered a number of EEG data quality and event morphology indicators as possible explanatory factors for the degree of concordance of the cESI-related GLM results.

First, we considered characteristics of the EEG signal: number of IED, morphology and amplitude of IED, presence or absence of consecutive slow wave or focal slowing of EEG background. Second, we considered possible contamination of the scalp EEG signal and consequently in the source signal as a result of incomplete correction of the pulse-related artefact, by calculating the proportion of QRS complexes in which artefact correction failed. Third, we calculated the correlation between $\mathrm{cESI}$ and $\mathrm{cESI}_{\mathrm{c}}$ (after convolution with HRF) to estimate the spatial specificity of cESI signal.

Difference between the group of concordant cases $(\mathrm{C}$ and $\mathrm{C}+)$ and the group of discordant cases D were tested with Mann-Whitney non-parametric tests for independent samples. 


\section{RESULTS}

\section{Concordance and clinical relevance:}

The results of ESI and SPM\{F\} BOLD maps are summarised in Table 2.

In total, 17 types of IED were identified. ESI was of poor quality in 2/17 IED types (polyspikes with low signal to noise of average IED and instable ESI) so that 15 IED types were considered for further analysis. ESI was spatially concordant with electro-clinical data in 13/15 IED types and with 7 intracranial EEG studies (4 patients). The 2 discordant cases (patient 4 , IED types $4 \mathrm{a}, 4 \mathrm{~b}$ ) are discussed in detail below.

In 14/15 IED types we observed significant BOLD signal changes related to all epileptic activity (combined cESI+IED SPM $\{F\}$ maps). In 10 of these IED types, the pattern of BOLD signal changes was concordant $(\mathrm{C}, \mathrm{C}+)$ with electro-clinical localisation of the irritative zone and structural lesion. Clusters were also found in regions concordant with the DMN in 4 of these 10 cases. A significant amount of BOLD variance was attributed to the cESI regressor alone in 7 of the 10 concordant cases (Figure 2 and 3) and in these 7 cases the most statistically significant clusters (outside the DMN) were concordant with electro-clinical and structural imaging data. Significant cESI-related or cESI+IED-related BOLD changes (cESI model) were concordant with the IED-related results (IED model), in all cases except case 4a/b (see below). In the 7 IED types (1, 4a, 4b, 5, 6a, 6b, 6c: 4 patients) in whom intracranial EEG was available, the use of cESI model clearly increased the size of the concordant clusters in 4/7 and showed similar results to those obtained with the IED model in the 3/7 remaining cases.

In 4 IED types (7, 9a,b,c: 2 patients), a pattern of bilateral, diffuse cESI-related BOLD signal changes was found, affecting all lobes particularly around large vessels (interhemispheric fissure, sylvian fissure, around brainstem (Figures 5 and 6). These spatial maps are similar to spatial maps obtained by mapping BOLD changes related to motion or cardiac (pulserelated) confounds.

In 1/15 IED type (patient 6c, 10 IED), no significant cESI or cESI+IED-related BOLD change was revealed.

$\underline{\text { Illustrative case reports: }}$ 
In patient 1 (right cryptogenic TLE), the SPM\{F\} map for combined cESI+IED revealed BOLD clusters in the right hippocampus, and regions of the DMN (Figure 2). This was concordant with intracranial EEG, which showed a majority of IED originating from the right hippocampus with independent or synchronous lateral temporal IED, explaining the localisation of ESI in the right lateral temporal cortex. By comparison, the $\operatorname{SPM}\{F\}$ map for IED in the simpler model showed only very small clusters in the right cingulate cortex and the right hippocampus.

In patient 4 (left occipital focal cortical dysplasia, bilateral anterior temporal IED), the epileptogenic zone was presumed to be localised in the left occipital lobe because the seizures were characterised by a visual aura and a focal cortical dysplasia was evident on structural MRI. However, interictal EEG showed left and right anterior temporal IED (leftsided predominance; 88\%) and, ESI showed left and right anterior temporal sources. BOLD changes related to left-sided anterior temporal cESI were revealed in the left occipital region, concordant with the MRI lesion (Figure 3). BOLD signal change related to right temporal cESI+IED were also found in the left occipital and temporal regions. Subsequent intracranial EEG recording confirmed a left occipital epileptogenic zone and the patient is seizure free with residual auras after left occipital resection. In contrast, for the IED model, there was no significant IED-related BOLD change in the left occipital or temporal cortex.

In patient 5 (right frontal epilepsy): SPM\{F\} maps for cESI showed bilateral medial and lateral frontal BOLD clusters with a right-sided predominance. Intracranial recording confirmed an extensive right frontal irritative zone predominating in the medial region. The patient continues to have seizures despite a limited right anterior frontal resection that did not include the strongest cESI BOLD cluster. The cESI model showed a larger BOLD cluster in the medial frontal cortex, than did the IED model, with more discrete involvement of the DMN (Figure 4).

In the other concordant patients, no intracranial EEG recording was performed: In patient 2, maps obtained with the cESI model showed only a left temporal BOLD cluster compared to a more widespread map of BOLD clusters obtained with the IED model (left temporal, left insula, DMN). In patient 3, results of cESI model and IED model showed similar spatial 
maps. In patient $6(6 a, 6 b, 6 c)$, results were similar with both cESI- and IED-models; $6 \mathrm{c}$ showed no significant BOLD changes with either model. In patients 8 and 10, multifocal BOLD clusters included right and left frontal clusters respectively, which were concordant with electro-clinical data and ESI.

\section{Factors that influence the degree of concordance:}

The discordant group generally showed diffuse bilateral cortical changes which presumably reflected contamination of cESI by artefacts (Table 2). The number of IED was significantly lower in the discordant (D) group than in the concordant $(C / C+)$ group (median 34 vs. 193, $Z=2.83, p=0.002$ ). There was no group difference in morphology of IED, presence of subsequent slow wave or focal slowing of EEG background. There was no difference between the groups regarding correlation of the signal derived from the IED source and the control region (median 0.85 vs. $0.76, Z=1.00, p=0.32$ ). Looking at the variance explained by confounds modelling motion or pulse artefacts, we found that the BOLD changes associated with the cardiac confounds also affected the perisylvian areas whereas the BOLD changes associated with motion showed a diffuse cortical localisation. The analysis of the quality of the QRS labelling on the ECG for correction of pulse-related artefacts showed that there was a greater percentage of missed R-peak markers in the discordant group (median $2.00 \%$ vs. $0.08 \%$, Mann-Whitney test $Z=2.00, p=0.046)$ (Table 3). 


\section{DISCUSSION}

Using continuous ESI (CESI) of the source of IED as a parametric model for BOLD signal changes, we showed that:

1) In models that also included IED-based effects, cESI explained significant amounts of additional variance of the BOLD signal in 14/15 IED studies;

2) There was generally good spatial concordance between the cESI-related BOLD changes with the presumed localisation of the epileptic focus suggested by structural imaging and electro-clinical data. cESI-correlated changes were also found distant from the region of IED onset, mostly in regions known to be part of the DMN (Aghakhani, et al. 2006; Hamandi, et al. 2006; Laufs, et al. 2006).

Discordant cases had fewer IED and cESI was probably contaminated by uncorrected artefacts in these cases.

These observations suggest that, in most cases studied here, cESI can reflect the activity of the epileptic focus rather than mostly independent fluctuations in background activity. Its inclusion as a predictor of BOLD changes in GLM could lead to improved mapping of the BOLD changes associated with interictal epileptic activity in selected cases with frequent IED.

\section{Methodological considerations}

Our choice of continuous ESI as a parametric regressor to model the BOLD signal was motivated by the following considerations:

1) EEG-fMRI has low sensitivity, predominantly due to the requirement for a significant number of clearly visible scalp IEDs;

2) Simultaneous scalp and intracranial studies have shown that IED visible on scalp EEG usually involve at least $6-10 \mathrm{~cm}^{2}$ of excited cortex (Tao, et al. 2005) and that as little as 1:2000 IED recorded by intracranial electrodes can be visually detected with scalp electrodes (Alarcon, et al. 1994).

3) Projection of the EEG signal in source space can allow the detection of additional events for GLM analysis, increasing the statistical power of the analysis (Liston, et al. 2006a). 
Projection of the signal into source space offers an elegant spatial filter for the EEG signal and the resultant source signal is more robust to artefact contamination than the EEG signal (Brookes, et al. 2009).

4) Two recent studies have validated the use of ESI performed on the averaged IED detected in the MR scanner during fMRI acquisition results for the localisation of the IED onset (Groening, et al. 2009; Vulliemoz, et al. 2009).

5) Taking the amplitude of IED into consideration rather than using a one-size-fits-all approach such as in the "event-related" approach to fMRI data analysis (IED model) used in this study might improve the modelling of BOLD changes.

The proposed approach represents a more quantitative EEG-based modelling strategy for BOLD signal fluctuations related to epileptic activity than the approach based on visual identification of IED, categorisation and representation as stick functions. In contrast to the method described by Liston et al (Liston, et al. 2006a), we did not limit the additional projected source-derived regressor to epochs corresponding to a specific waveform, but instead wanted to assess the BOLD correlates of the entire recording, while accounting to various sources of noise in the GLM. Another approach would be to parameterise the visually-identified IED; indeed our results suggest such an approach would probably benefit the IED-based model. In terms of the range of strategies available for the analysis of simultaneously acquired EEG-fMRI data in patients with epilepsy, our study can be positioned between visual IED-based GLM and data-driven approaches such as ICA of the EEG (Jann, et al. 2008; Marques, et al. 2009) or fMRI (Rodionov, et al. 2007). While the ICA approaches are potentially superior for identifying artefacts in the EEG signal, the selection of an "epileptic independent component" remains subjective and hence components containing more subtle activity may not be recognised. In that respect, the value of $\mathrm{cESI}$ is that it contains all activity including the one which can be potentially left out by ICA. The ESI solution varies smoothly across solution points and, therefore, the cESI regressor is not strongly influenced by the inherent uncertainty of source localisation. Conversely, this lack of spatial specificity means that the regressor is likely to incorporate activity remote from 
the true generator. This is one of the reasons for the inclusion of a control cESI regressor in the model.

Along with these potentially beneficial factors, this more user-independent analysis in source space carries significant challenges. In the spontaneous EEG, IED account only for a small proportion of the data and the Signal to Noise Ratio (SNR) is much lower than for individual or averaged IED, causing the cESI to be contaminated by noise. By first defining a region of interest based on ESI of averaged IED, we maximised the epileptic contribution in cESI. We postulated that the underlying ongoing epileptic activity, as recorded from intracranial recordings in similar patients, would be reflected in the source signal despite some contamination with noise. Electrode motion- and myogenic artefacts contaminating the scalp EEG are projected into source space, corrupting the ESI signal. Recent ESI simulation and experimental studies showed that the signal to noise ratio was increased through projection in source space as interference is correlated across EEG channels (Brookes, et al. 2008). The authors suggested that the same benefit should apply to other ESI strategies, notably distributed linear inverse solution as used in our study. Visual inspection of the scalp EEG and cESI signal, however, revealed residual artefacts surviving the projection in source space. Therefore, we used additional «scan nulling » regressors (Lemieux, et al. 2007) to account for the variance of the BOLD signal in the volumes acquired during these time intervals. Additionally, despite a conservative analysis strategy to remove variance caused by motion and cardiac pulsations, we found consistent results in the discordant cases that were strongly suggestive of residual artefacts as shown by spatial similarity with the map of signal changes related to cardiac confounds (as modelled by the linear «Time After Pulse » methods (Liston, et al. 2006b)) and motion confounds (Figures 5 and 6). We found that the pulse-artefact correction was significantly better in concordant cases. The effect of cardiac cycle variability (Shmueli, et al. 2007) and other non-linear cardiac effects (Wan, et al. 2006) or respiratory effects (Birn, et al. 2006) might help explain this residual contamination. We were not able to assess the residual pulse-related activity by formal comparison of the low frequency content of EEG spectrum inside/outside the MR scanner due to the different levels of arousal between the two recordings in most patients. 
Moreover, the discordant cases had significantly fewer IED, and therefore a lower SNR of the epileptic activity in cESI and an increased effect of residual confounds. Cases with fewer than 40 IED were all discordant and all cases with concordant BOLD cluster related to cESI had more than 100 IED.

Different EEG frequency bands have been associated with distinct haemodynamic spatial signatures in healthy subjects (Laufs, et al. 2003; Mantini, et al. 2007). A future refinement of our analysis strategy will be to decompose the source signal in separate frequency bands to build parametric regressors. This would allow us to be more specific about the BOLD changes associated with source activity at different frequencies.

\section{Neurophysiological and clinical relevance}

Our results suggest that the use of continuous ESI as a parametric regressor for EEG-fMRI analysis can improve the modelling of interictal BOLD fluctuations in focal epilepsy. In 7 IED types (4 patients) with concordant results, intracranial recording was available as a gold standard for localising the IED generators. Compared with the conventional IED model, we found that cESI model revealed a new or larger concordant BOLD cluster in 4/7 cases.

In most patients, we found good spatial concordance between cESI-related BOLD changes and non-invasive localisation of the epileptogenic focus (lesion on structural MRI, electroclinical data). This suggests that the cESI signal reflects the activity of the epileptic focus. Although the epileptic activity may interact with the physiological activity of resting state networks (as illustrated by DMN BOLD clusters in 4/10 concordant cases), we would expect a different spatial distribution of BOLD signal changes if the cESI signal was dominated by non-epileptic activity (systematic involvement of resting state networks, absence of clusters localising the epileptogenic focus).

Case $4 \mathrm{a}$ is particularly instructive from a clinical perspective. Occipital BOLD changes were observed in relation to anterior temporal IED and anterior temporal ESI, probably reflecting IED propagation along occipito-temporal pathways (Aykut-Bingol and Spencer 1999). A left occipital epileptogenic zone was identified by subsequent subdural EEG recording. The BOLD changes appeared to take place at the epileptogenic zone, « upstream » from bilateral 
anterior temporal IED, which reflect propagation areas and an irritative zone with a much larger extent than the epileptogenic zone. This effect may be related to BOLD changes preceding IED as has been shown in some cases of focal epilepsy, mostly in relation to generalised IED (Hawco, et al. 2007; Jacobs, et al. 2009; Lemieux, et al. 2008; Vaudano, et al. 2009). Other studies rather suggested a "downstream" effect, with IED modulating BOLD signal and presumably neuronal activity in distant regions involved in the epileptogenic network, such as the DMN (Kobayashi, et al. 2006b; Laufs, et al. 2006; Salek-Haddadi, et al. 2006) and the limbic network (Kobayashi, et al. 2006a) in medial temporal epilepsy. One hypothesis to explain this neurovascular uncoupling might be that high frequency activity (frequently associated with focal cortical dysplasia) or closed electric field generated by interneurons was timelocked to the IED but was not detected in our scalp recording. This activity could have more hemodynamical correlates than the propagated temporal activity that was however, easier to detect on the scalp. Another hypothesis could be that distorsion and drop out known to affect the inferior temporal lobes prevented the observation of temporal BOLD changes related to the temporal IED.

The availability of invasive EEG recording and post-operative outcome data in larger patient groups will allow us to further validate the proposed method.

\section{Conclusion}

Continuous EEG source imaging could improve the modelling of the BOLD signal in interictal studies of patients with focal epilepsy. This could enhance our ability to localise the irritative zone in selected pre-surgical epilepsy cases using EEG-fMRI. We have identified specific features (number of IED, entropy of IED, low residual pulse-related artefact) which might be helpful to select patients in whom our model would be more likely to have clinical relevance. Validation and assessment of gains in sensitivity and specificity of the method need to be addressed in larger groups of patients. 


\section{REFERENCES}

Aghakhani Y, Kobayashi E, Bagshaw AP, Hawco C, Benar CG, Dubeau F, Gotman J. (2006): Cortical and thalamic fMRI responses in partial epilepsy with focal and bilateral synchronous spikes. Clin Neurophysiol 117(1):177-91.

Al-Asmi A, Benar CG, Gross DW, Khani YA, Andermann F, Pike B, Dubeau F, Gotman J. (2003): fMRI activation in continuous and spike-triggered EEG-fMRI studies of epileptic spikes. Epilepsia 44(10):1328-39.

Alarcon G, Guy CN, Binnie CD, Walker SR, Elwes RD, Polkey CE. (1994): Intracerebral propagation of interictal activity in partial epilepsy: implications for source localisation. J Neurol Neurosurg Psychiatry 57(4):435-49.

Allen PJ, Josephs O, Turner R. (2000): A method for removing imaging artifact from continuous EEG recorded during functional MRI. Neuroimage 12(2):230-9.

Allen PJ, Polizzi G, Krakow K, Fish DR, Lemieux L. (1998): Identification of EEG events in the MR scanner: the problem of pulse artifact and a method for its subtraction. Neuroimage 8(3):229-39.

Aykut-Bingol C, Spencer SS. (1999): Nontumoral occipitotemporal epilepsy: localizing findings and surgical outcome. Ann Neurol 46(6):894-900.

Bagshaw AP, Hawco C, Benar CG, Kobayashi E, Aghakhani Y, Dubeau F, Pike GB, Gotman J. (2005): Analysis of the EEG-fMRI response to prolonged bursts of interictal epileptiform activity. Neuroimage 24(4):1099-112.

Benar CG, Grova C, Kobayashi E, Bagshaw AP, Aghakhani Y, Dubeau F, Gotman J. (2006): EEG-fMRI of epileptic spikes: concordance with EEG source localization and intracranial EEG. Neuroimage 30(4):1161-70.

Birn RM, Diamond JB, Smith MA, Bandettini PA. (2006): Separating respiratory-variationrelated fluctuations from neuronal-activity-related fluctuations in $\mathrm{fMRI}$. Neuroimage 31(4):1536-48.

Brookes MJ, Mullinger KJ, Stevenson CM, Morris PG, Bowtell R. (2008): Simultaneous EEG source localisation and artifact rejection during concurrent $\mathrm{fMRI}$ by means of spatial filtering. Neuroimage 40(3):1090-104.

Brookes MJ, Vrba J, Mullinger KJ, Geirsdottir GB, Yan WX, Stevenson CM, Bowtell R, Morris PG. (2009): Source localisation in concurrent EEG/fMRI: applications at 7T. Neuroimage 45(2):440-52.

Friston KJ, Williams S, Howard R, Frackowiak RS, Turner R. (1996): Movement-related effects in fMRI time-series. Magn Reson Med 35(3):346-55.

Grave de Peralta Menendez R, Gonzalez Andino S, Lantz G, Michel CM, Landis T. (2001): Noninvasive localization of electromagnetic epileptic activity. I. Method descriptions and simulations. Brain Topogr 14(2):131-7.

Groening K, Brodbeck V, Moeller F, Wolff S, van Baalen A, Michel CM, Jansen O, Boor R, Wiegand G, Stephani U and others. (2009): Combination of EEG-fMRI and EEG source analysis improves interpretation of spike-associated activation networks in paediatric pharmacoresistant focal epilepsies. Neuroimage 46(3):827-33.

Hamandi K, Salek-Haddadi A, Laufs H, Liston A, Friston K, Fish DR, Duncan JS, Lemieux L. (2006): EEG-fMRI of idiopathic and secondarily generalized epilepsies. Neuroimage 31(4):1700-10.

Hawco CS, Bagshaw AP, Lu Y, Dubeau F, Gotman J. (2007): BOLD changes occur prior to epileptic spikes seen on scalp EEG. Neuroimage 35(4):1450-8.

Jacobs J, Levan P, Moeller F, Boor R, Stephani U, Gotman J, Siniatchkin M. (2009): Hemodynamic changes preceding the interictal EEG spike in patients with focal epilepsy investigated using simultaneous EEG-fMRI. Neuroimage 45(4):1220-31.

Jann K, Wiest R, Hauf M, Meyer K, Boesch C, Mathis J, Schroth G, Dierks T, Koenig T. (2008): BOLD correlates of continuously fluctuating epileptic activity isolated by independent component analysis. Neuroimage 42(2):635-48.

Kobayashi E, Bagshaw AP, Benar CG, Aghakhani Y, Andermann F, Dubeau F, Gotman J. (2006a): Temporal and extratemporal BOLD responses to temporal lobe interictal spikes. Epilepsia 47(2):343-54.

Kobayashi E, Bagshaw AP, Grova C, Dubeau F, Gotman J. (2006b): Negative BOLD responses to epileptic spikes. Hum Brain Mapp 27(6):488-97. 
Krakow K, Woermann FG, Symms MR, Allen PJ, Lemieux L, Barker GJ, Duncan JS, Fish DR. (1999): EEG-triggered functional MRI of interictal epileptiform activity in patients with partial seizures. Brain 122 (Pt 9):1679-88.

Lantz G, Spinelli L, Seeck M, de Peralta Menendez RG, Sottas CC, Michel CM. (2003): Propagation of interictal epileptiform activity can lead to erroneous source localizations: a 128-channel EEG mapping study. J Clin Neurophysiol 20(5):311-9.

Laufs H, Hamandi K, Salek-Haddadi A, Kleinschmidt AK, Duncan JS, Lemieux L. (2006): Temporal lobe interictal epileptic discharges affect cerebral activity in "default mode" brain regions. Hum Brain Mapp.

Laufs H, Krakow K, Sterzer P, Eger E, Beyerle A, Salek-Haddadi A, Kleinschmidt A. (2003): Electroencephalographic signatures of attentional and cognitive default modes in spontaneous brain activity fluctuations at rest. Proc Natl Acad Sci U S A 100(19):11053-8.

Lemieux L, Laufs H, Carmichael D, Paul JS, Walker MC, Duncan JS. (2008): Noncanonical spike-related BOLD responses in focal epilepsy. Hum Brain Mapp 29(3):329-45.

Lemieux L, Salek-Haddadi A, Lund TE, Laufs H, Carmichael D. (2007): Modelling large motion events in $\mathrm{fMRI}$ studies of patients with epilepsy. Magn Reson Imaging 25(6):894-901.

Liston AD, De Munck JC, Hamandi K, Laufs H, Ossenblok P, Duncan JS, Lemieux L. (2006a): Analysis of EEG-fMRI data in focal epilepsy based on automated spike classification and Signal Space Projection. Neuroimage 31(3):1015-24.

Liston AD, Lund TE, Salek-Haddadi A, Hamandi K, Friston KJ, Lemieux L. (2006b): Modelling cardiac signal as a confound in EEG-fMRI and its application in focal epilepsy studies. Neuroimage 30(3):827-34.

Mantini D, Perrucci MG, Del Gratta C, Romani GL, Corbetta M. (2007): Electrophysiological signatures of resting state networks in the human brain. Proc Natl Acad Sci U S A 104(32):13170-5.

Marques JP, Rebola J, Figueiredo P, Pinto A, Sales F, Castelo-Branco M. (2009): ICA decomposition of EEG signal for $f M R I$ processing in epilepsy. Hum Brain Mapp.

Pasqual Marqui RD, Sekihara K, Brandeis D, Michel CM. 2009. Imaging the electrical neuronal generators of EEG/MEG. In: Michel CM KT, Brandeis D, Gianotti LRR, Wackermann J, editor. Electrical Neuroimaging. Cambridge: Cambridge University Press. p 49-77.

Raichle ME, MacLeod AM, Snyder AZ, Powers WJ, Gusnard DA, Shulman GL. (2001): A default mode of brain function. Proc Natl Acad Sci U S A 98(2):676-82.

Rodionov R, De Martino F, Laufs H, Carmichael DW, Formisano E, Walker M, Duncan JS, Lemieux L. (2007): Independent component analysis of interictal fMRI in focal epilepsy: comparison with general linear model-based EEG-correlated fMRI. Neuroimage 38(3):488-500.

Salek-Haddadi A, Diehl B, Hamandi K, Merschhemke M, Liston A, Friston K, Duncan JS, Fish DR, Lemieux L. (2006): Hemodynamic correlates of epileptiform discharges: an EEG-fMRI study of 63 patients with focal epilepsy. Brain Res 1088(1):148-66.

Shmueli K, van Gelderen P, de Zwart JA, Horovitz SG, Fukunaga M, Jansma JM, Duyn JH. (2007): Low-frequency fluctuations in the cardiac rate as a source of variance in the resting-state fMRI BOLD signal. Neuroimage 38(2):306-20.

Sillanpaa M. (2000): Long-term outcome of epilepsy. Epileptic Disord 2(2):79-88.

Spinelli L, Andino SG, Lantz G, Seeck M, Michel CM. (2000): Electromagnetic inverse solutions in anatomically constrained spherical head models. Brain Topogr 13(2):11525.

Tao JX, Ray A, Hawes-Ebersole S, Ebersole JS. (2005): Intracranial EEG substrates of scalp EEG interictal spikes. Epilepsia 46(5):669-76.

Vaudano AE, Laufs H, Kiebel S, Carmichael DW, Hamandi K, Guye M, Thornton R, Rodionov R, Friston KJ, Duncan JS and others. (2009): Causal Hierarchy within the Thalamo-Cortical Network in Spike and Wave Discharges. PLoSone.

Vulliemoz S, Thornton R, Rodionov R, Carmichael DW, Guye M, Lhatoo S, McEvoy AW, Spinelli L, Michel CM, Duncan JS and others. (2009): The spatio-temporal mapping of epileptic networks: combination of EEG-fMRI and EEG source imaging. Neuroimage 46(3):834-43. 
Wan X, Iwata K, Riera J, Ozaki T, Kitamura M, Kawashima R. (2006): Artifact reduction for EEG/fMRI recording: nonlinear reduction of ballistocardiogram artifacts. Clin Neurophysiol 117(3):668-80. 


\section{LEGENDS}

Figure 1 : Summary of the analysis strategy : a) IED are marked on EEG recording; b) IED are averaged; c) Electric Source Imaging is performed at averaged IED onset (ESI); d) continuous ESI: cESI is extracted for the entire EEG in the region of ESlo; e) the signal is convolved with the Hemodynamic Response Function; f) the result of convolution is downsampled to the scanner repetition time $(\mathrm{TR}) ; \mathrm{g}$ ) the resulting vector is incorporated as a regressor into the General Linear Model for fMRI analysis.

Figure 2 : Case 1: Scalp EEG, ESI, cESI- and IED-related BOLD changes (SPM\{F\} maps, family-wise error corrected $\mathrm{p}<0.05)$ : a) Right temporal and temporo-occipital IED (black circles); b) result of ESI showing right lateral temporal source; c) cESI model: combined cESI+IED SPM\{F\} maps: clear anatomical localisation in the right hippocampus with additional regions of the Default Mode Network (DMN); d) IED model: IED-related BOLD changes: very small BOLD cluster in the right anterior cingulate gyrus (maximum) and right hippocampus. Intracranial EEG confirmed right temporal medial and lateral IED with predominance in the medial structures. The 'glass brain' SPM $\{F\}$ maps were normalised for display purposes only; the overlay on T1-weighted image is in individual space.

Figure 3 : Case 4a : Scalp EEG, ESI, cESI- and IED-related BOLD changes (all are SPM\{F\} maps, family-wise error corrected $p<0.05)$ : a) scalp EEG showing left anterior temporal IED (black circles); b) result of ESI showing left anterior temporal source; c) left occipital FCD seen as BOLD increase in the white matter on coronal FLAIR image (white arrow); d) cESI model: cESI SPM\{F\} maps: left occipital BOLD cluster co-localised with MRI lesion; e) IED model: no significant IED-related BOLD change. Intracranial EEG with subdural electrodes confirmed a left occipital epileptogenic zone. The 'glass brain' $\operatorname{SPM}\{F\}$ maps were normalised for display purposes only; the overlay on T1-weighted images is in individual space. 
Figure 4 : Case 5: Scalp EEG, ESI, cESI- and IED-related BOLD changes (all are SPM\{F\} maps, family-wise error corrected $p<0.05)$ : a) Right frontal IED on scalp EEG (black circles); b) result of ESI showing right medial frontal source; c) cESI model: cESI-related BOLD changes: significant BOLD changes in the right medial frontal lobe, concordant with ESI. d) IED model: IED-related BOLD changes: Much smaller BOLD cluster in the anterior right medial frontal lobe (crossline) and predominance of frontal lateral clusters and DMN-related BOLD clusters. Intracranial EEG recording with depth electrodes confirmed the right medial frontal localisation. The 'glass brain' SPM $\{F\}$ maps were normalised for display purposes only; the overlay on T1-weighted images is in individual space.

Figure 5: Case 7: Scalp EEG, ESI and cESI-related BOLD changes (all are SPM\{F\} maps, family-wise error corrected $p<0.05)$ : a) Left temporal IED (black circles); b) result of ESI showing left inferior temporal source; c) cESI model: cESI-related BOLD changes: diffuse bilateral BOLD changes affecting all lobes with very high statistical value in regions of large vessels (superior midline, sylvian fissure, around brainstem) and white matter with spatial similarity to the SPF $\{F\}$ map of cardiac confounds suggesting residual cardiac artefact (white arrows); d) motion-related BOLD changes e) cardiac-related BOLD changes (white arrows show spatial similarities with cESI-related BOLD clusters).

Figure 6: Case 9a: Scalp EEG, ESI and cESI- related BOLD changes (all are family-wise error corrected $p<0.05)$ : a) Left fronto-temporal IED (black circles), b) result of ESI showing left anterior lateral temporal source; c) cESI model: cESI-related BOLD changes: diffuse bilateral BOLD changes affecting all lobes, with very high statistical value in regions of large vessels (superior midline, sylvian fissure, around brainstem) with spatial similarity to the SPF $\{F\}$ map of cardiac and motion confounds suggesting residual motion and cardiac artefact (white arrows); d) motion-related BOLD changes; e) cardiac-related BOLD changes (white arrows show spatial similarities with cESI-related BOLD clusters). 


\begin{tabular}{|c|c|c|c|c|c|c|}
\hline $\begin{array}{l}\text { IED } \\
\text { type }\end{array}$ & $\begin{array}{l}\text { Gender, } \\
\text { age }\end{array}$ & Seizure semiology & MRI & $\begin{array}{l}\text { IED focus } \\
\text { (scalp EEG) }\end{array}$ & $\begin{array}{c}\text { Number of IED } \\
\text { during fMRI } \\
\text { (Number of 20- } \\
\text { min fMRI } \\
\text { sessions) }\end{array}$ & $\begin{array}{c}\text { Intracranial } \\
\text { EEG }\end{array}$ \\
\hline 1 & M, 31y. & $\begin{array}{c}\text { Bizzare vague } \\
\text { sensation, } \\
\text { CPS with } \\
\text { dystonic posture L } \\
\text { hand }\end{array}$ & $\mathrm{N}$ & R T & $189(2)$ & $\mathrm{R} T$ lat post \\
\hline 2 & M, 30y. & $\begin{array}{l}\text { Aphasic SPS } \\
\text { No aura, CPS }\end{array}$ & $\mathrm{N}$ & L T & $161(2)$ & na \\
\hline 3 & $F, 48 y$. & $\begin{array}{c}\text { No aura, } \\
\text { CPS with oral and } \\
\text { manual } \\
\text { automatisms } \\
\end{array}$ & $\mathrm{N}$ & $\mathrm{L} T$ & $197(2)$ & na \\
\hline $\begin{array}{l}4 a \\
4 b\end{array}$ & M, 21y. & $\begin{array}{c}\text { CPS, oral } \\
\text { automatisms, R } \\
\text { clonic jerks, SGS }\end{array}$ & $\begin{array}{l}\text { FCD L } \\
\text { medial } \\
\text { occipital }\end{array}$ & $\begin{array}{l}\mathrm{LT} \\
\mathrm{RT}\end{array}$ & $\begin{array}{l}312(2) \\
56(2)\end{array}$ & L Occ medial \\
\hline 5 & M, 27y. & $\begin{array}{c}\text { Cloni R face/arm } \\
\quad->\text { SGS }\end{array}$ & $\mathrm{N}$ & R F & $2239(2)$ & R Fmedial ant \\
\hline $6 a$ & M, 19y. & $\begin{array}{l}\text { Epilepsia partialis } \\
\text { continua L leq }\end{array}$ & $\mathrm{N}$ & $\begin{array}{l}\text { Central } \\
\text { midline }\end{array}$ & $206(3)$ & $\mathrm{R}$ medial F-Par \\
\hline $6 b$ & IVI, 10y. & --> rare SGS & & R F-central & $121(3)$ & \\
\hline $6 c$ & & & & R F & $10(3)$ & \\
\hline 7 & M, & $\begin{array}{c}\text { Olfactory-gustatory } \\
\text { aura, CPS }\end{array}$ & L HS & $\mathrm{L} T$ & $33(2)$ & na \\
\hline $\begin{array}{l}8 a \\
(8 b)\end{array}$ & M, 22y. & $\begin{array}{l}\text { No aura, } \\
\text { CPS with } L \text { head } \\
\text { version }\end{array}$ & $\mathrm{N}$ & $\begin{array}{c}\text { Bil F } \\
\text { Bil F } \\
\text { polyspikes\# }\end{array}$ & $\begin{array}{l}269(3) \\
101(3)\end{array}$ & na \\
\hline $\begin{array}{l}9 a \\
9 b \\
9 c\end{array}$ & M, 25y. & $\begin{array}{c}\text { Epigastric , } \\
\text { auditory, gustatory } \\
\text { or } \\
\text { heautoscopic aura, } \\
\text { tonic posture R } \\
\text { hand }\end{array}$ & $\mathrm{N}$ & $\begin{array}{l}\text { L F-T } \\
\text { L F polar } \\
\text { L Par }\end{array}$ & $\begin{array}{l}38(3) \\
35(3) \\
23(3)\end{array}$ & na \\
\hline $10 a$ & F, 37y. & No aura, CPS & $\begin{array}{l}\text { L F white } \\
\text { matter lesion }\end{array}$ & $\underset{L>R}{L F \text { and Bil F }}$ & $72(2)$ & na \\
\hline (10b) & & & & R F\# & $11(2)$ & \\
\hline
\end{tabular}

Table 1: Clinical, electrophysiological and imaging data: Clinical, EEG and radiological data. R/L/Bil: right/left/bilateral; T: temporal; F: frontal; Par: parietal; Occ: occipital; post: posterior; SPS/CPS : Simple/Complex Partial Seizure, SGS : Secondarily Generalised tonicclonic Seizure, FCD : Focal Cortical Dysplasia, HS : Hippocampal Sclerosis. \# not considered for further analysis as good quality ESI was not possible, see text). 


\begin{tabular}{|c|c|c|c|c|c|c|c|}
\hline $\begin{array}{l}\text { Code } \\
\text { of the } \\
\text { IED } \\
\text { type }\end{array}$ & $\begin{array}{l}\text { Scalp } \\
\text { EEG } \\
\text { focus }\end{array}$ & ESI & $\begin{array}{l}\text { Conventional IED model } \\
\text { F(IED) (FWE) }\end{array}$ & $\begin{array}{l}\text { EEG source activity: } \\
\text { F(cESI) (FWE) }\end{array}$ & $\begin{array}{l}\text { metric cESI model } \\
\\
\text { All epileptic activity } \\
\text { (EEG source and IED } \\
\text { F(cESI+IED) (FWE) } \\
\text { Only additional clusters to } \\
\text { cESI column on the left }\end{array}$ & $\begin{array}{l}\text { Concordance } \\
\mathrm{cESI} / \mathrm{cESI}+\mathrm{IED}\end{array}$ & $\begin{array}{l}\text { Additional information } \\
\text { obtained from cESI+IED model } \\
\text { compared to IED model } \\
\text { (icEEG as gold standard) }\end{array}$ \\
\hline 1 & $\mathrm{RT}$ & RTlat & RCing ant, RTmes & Bil Pmes*, Bil TP*, RFmes & + RTmes & $\mathrm{D} / \mathrm{C}+$ & Larger cluster $\mathbf{R}$ hippocampus \\
\hline 2 & LT & LTlat & $\begin{array}{c}\text { Bil Pmes* }{ }^{*} \text { LTsup, Linsul, RTsup, } \\
\text { LTP }\end{array}$ & n.s. & + LTinf & $--/ C$ & n.a. \\
\hline 3 & LT & LTlat & $\begin{array}{c}\text { Bil Pmes*, LTP*, Linsul, LTlat, } \\
\text { LTmes, Rcereb }\end{array}$ & $\begin{array}{l}\text { Bil Pmes*, LTmes, LTP*, } \\
\text { LTlat, Linsul, LF }\end{array}$ & (idem cESI) & $\mathrm{C}+/ \mathrm{C}+$ & n.a. \\
\hline $4 a$ & LT & LTant & n.s. & LOmes & (idem cESI) & $\mathrm{C} / \mathrm{C}$ & New L occipital cluster \\
\hline $4 b$ & RT & RTpol & RTP & n.s. & $\begin{array}{l}+ \text { LOmes } \\
+ \text { LTinf }\end{array}$ & $--/ C$ & New L occipital cluster \\
\hline 5 & RF & RForb & $\begin{array}{l}\text { Bil TP*, Bil Pmes*, RFmes, Bil } \\
\text { Thal, RFoperc }\end{array}$ & $\begin{array}{l}\text { Bil Thal, RSMA, RFmes, Bil } \\
\text { TP*, Bil Pmes*, R>>L Flat }^{*} \text {, }\end{array}$ & (idem cESI) & $\mathrm{C}+/ \mathrm{C}+$ & Larger $\mathbf{R}$ medial frontal clusters \\
\hline $6 a$ & Vertex & $\begin{array}{l}\text { L>R central } \\
\text { mes }\end{array}$ & Rparacentral lobule & Rparacentral lobule, Lcereb & (idem cESI) & $\mathrm{C}+/ \mathrm{C}+$ & Similar, no additional information \\
\hline $6 b$ & R FC & RFClat & RFmid & RFmid, RTsup & (idem cESI) & $\mathrm{C}+/ \mathrm{C}+$ & Similar, no additional information \\
\hline $6 c$ & RF & RFlat & n.s. & n.s. & n.s. & $--/--$ & Similar, n.s. \\
\hline 7 & LT & LTmes & n.s. & $\begin{array}{c}\text { RFant (max) } \\
\text { Diffuse bilateral all lobes }\end{array}$ & (idem cESI) & $\mathrm{D} / \mathrm{D}$ & n.a. \\
\hline 8 & RF & RFant & 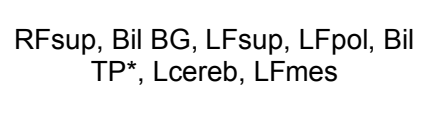 & $\begin{array}{l}\text { LFant, Bil Foperc, LTP*, Bil } \\
\text { Tsup, LPmes* }\end{array}$ & $\begin{array}{c}\text { + RFsup/mes, RTP*, Bil } \\
\text { Tlat, RPmes* }{ }^{*} \text {, LFant, R } \\
\text { Thal }\end{array}$ & $\mathrm{C}+/ \mathrm{C}+$ & n.a. \\
\hline $9 a$ & LFpol & LFpol & n.s. & $\begin{array}{l}\text { LFpost (max) } \\
\text { Diffuse bilateral all lobes }\end{array}$ & (idem cESI) & $\mathrm{D} / \mathrm{D}$ & n.a. \\
\hline $9 b$ & $\begin{array}{l}\text { LFinf } \\
\text { /LTant }\end{array}$ & LTlat & LTsup & $\begin{array}{l}\text { LFpost (max) } \\
\text { Diffuse bilateral all lobes }\end{array}$ & (idem cESI) & $\mathrm{D} / \mathrm{D}$ & n.a. \\
\hline $9 c$ & LP & LPlat & n.s. & $\begin{array}{l}\text { LFpost (max) } \\
\text { Diffuse bilateral all lobes }\end{array}$ & (idem cESI) & $\mathrm{D} / \mathrm{D}$ & n.a. \\
\hline 10 & $\mathrm{biF}$ & LFmid & $\begin{array}{l}\text { LFmid, Bil TP*, Bil Fmes, } \\
\text { LFoperc, RFmid }\end{array}$ & n.s. & $\begin{array}{c}\text { + ROmes, Bil TP*, } \\
\text { LFsup, LFmid, RCing ant, } \\
\text { LPmes*', LTlat }\end{array}$ & $--/ \mathrm{C}+$ & n.a. \\
\hline
\end{tabular}


Table 2: EEG/ESI results and results of SPM for parametric model and IED model. BOLD clusters are presented with decreasing statistical scores. ${ }^{*}$ Regions belonging to the Default Brain Network of the brain, not considered localising. R/L/Bil : Right/Left/Bilateral; F/P/T/O : Frontal/Parietal/Temporal/Occipital lobes ; SMA= Supplementary Motor Area ; FC : Fronto-Central ; Cing : Cingulate gyrus ; operc : opercular; Thal : Thalamus; WM: white matter; Ant/post/pol/mid: anterior/posterior/polar/middle, lat/med: lateral/medial. n.s.: non-significant; C/C+/D/--: Concordant/Concordant+/Discordant/no result available. Cluster smaller than 5 voxels were not reported in this Table except if they coincided with the statistical maximum or if no larger cluster was present. n.a.: not applicable (no intracranial EEG recording available). 


\begin{tabular}{|c|c|c|c|c|c|c|}
\hline $\begin{array}{l}\text { IED } \\
\text { type }\end{array}$ & $\begin{array}{l}\text { Scalp EEG } \\
\text { focus }\end{array}$ & ESI & $\begin{array}{c}\text { IED } \\
\text { during } \\
\text { fMRI } \\
\text { (fMRI } \\
\text { sessions) }\end{array}$ & $\begin{array}{l}\text { Correlation } \\
\text { cESI vs } \\
\text { cESI }\end{array}$ & $\begin{array}{c}\% R \\
\text { markers } \\
\text { missed }\end{array}$ & $\begin{array}{c}\text { cESI } \\
\text { Concordance } \\
\text { cESI/ cESI+IED }\end{array}$ \\
\hline 1 & RT & RTlat & $189(2)$ & 0.51 & 0.08 & $\mathrm{D} / \mathrm{C}+$ \\
\hline 2 & LT & LTlat & $161(2)$ & 0.86 & 0,04 & $--/ C$ \\
\hline 3 & LT & LTlat & $197(2)$ & 0.67 & 0.04 & $\mathrm{C}+/ \mathrm{C}+$ \\
\hline $4 a$ & LT & LTant & $312(2)$ & 0.95 & 0.05 & $\mathrm{C} / \mathrm{C}$ \\
\hline $4 b$ & RT & RTpol & $56(2)$ & 0.90 & 0.05 & $--/ C$ \\
\hline 5 & RF & RForb & $2239(2)$ & 0.28 & 0.12 & $\mathrm{C}+/ \mathrm{C}+$ \\
\hline $6 a$ & Vertex & $L>R$ central mes & $206(3)$ & 0.78 & $1.8 \#$ & $\mathrm{C}+/ \mathrm{C}+$ \\
\hline $6 b$ & R FC & RFClat & $121(3)$ & 0.88 & $1.8 \#$ & $\mathrm{C}+/ \mathrm{C}+$ \\
\hline $6 c$ & RF & RFlat & $10(3)$ & 0.79 & $1.8 \#$ & --/-- \\
\hline 7 & LT & LTmes & $33(2)$ & 0.85 & 0.66 & $D / D$ \\
\hline $8 a$ & RF & RFant & $370(3)$ & 0.52 & 3.80 & $\mathrm{C}+/ \mathrm{C}+$ \\
\hline $9 a$ & LFpol & LFpol & $38(3)$ & 0.85 & 2.00 & $\mathrm{D} / \mathrm{D}$ \\
\hline $9 b$ & LFinf/LTant & LTlat & $35(3)$ & 0.93 & 2.00 & $\mathrm{D} / \mathrm{D}$ \\
\hline $9 c$ & LP & LPlat & $23(3)$ & 0.83 & 2.00 & $\mathrm{D} / \mathrm{D}$ \\
\hline $10 a$ & $\mathrm{biF}$ & LFmid & $72(2)$ & 0.74 & 0.08 & $--/ C+$ \\
\hline
\end{tabular}

Table 3: Factors influencing the degree of concordance: EEG characteristics and results of SPM for IED-based GLM and parametric model (with control Region of Interest and additional scan nulling to remove artefact-affected EEG segments). \# persistent residual gradient artefact on ECG channel, but cardiac rhythm was very regular. R/L/Bil : Right/Left/Bilateral; F/P/T/O : Frontal/Parietal/Temporal/Occipital lobes; Ant/post/pol/mid : anterior/posterior/polar/middle, lat/med: lateral/medial. C/C+/D/--: Concordant/Concordant+/Discordant/no result available (See Table 2). 
Click here to download high resolution image

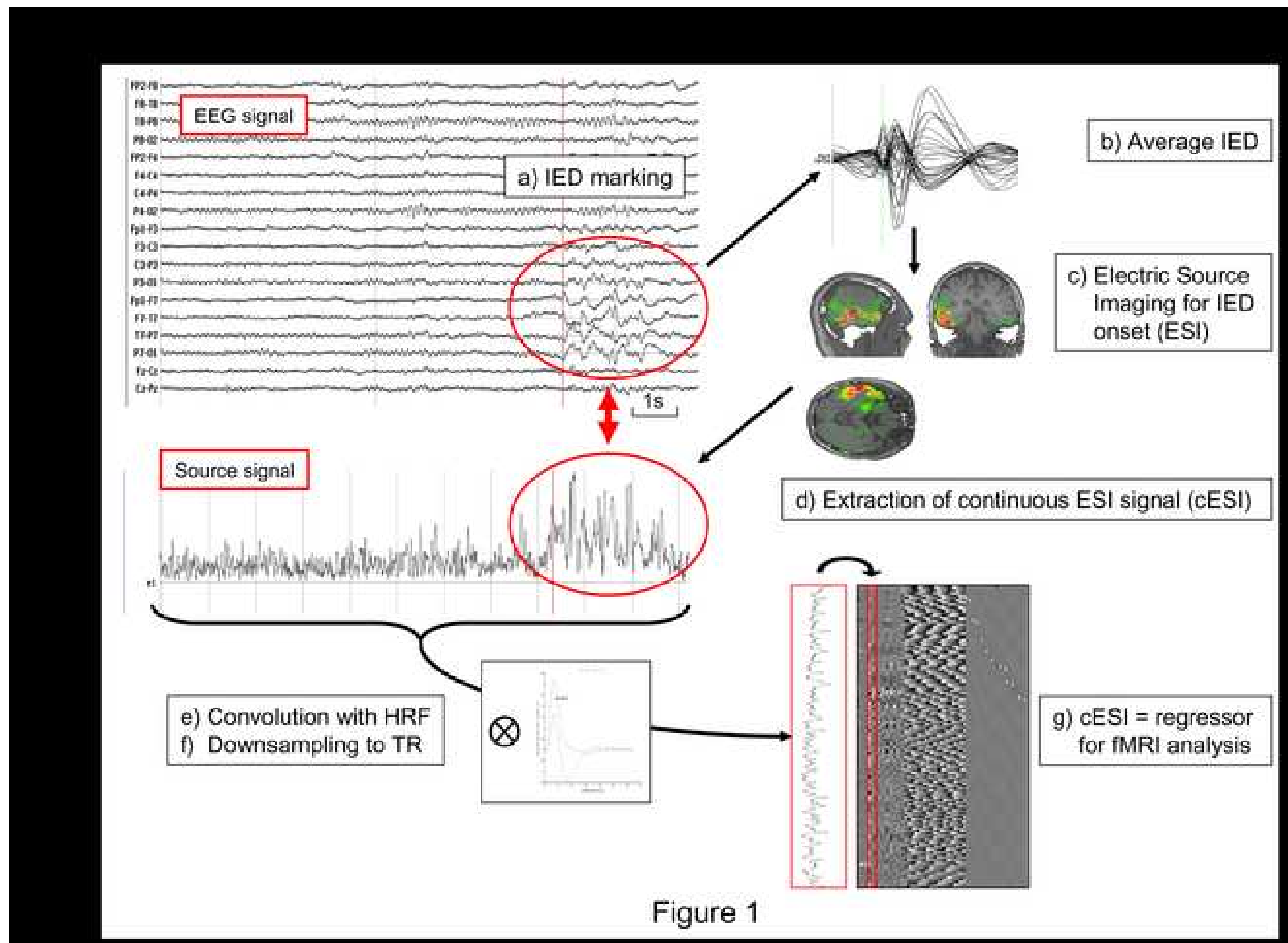



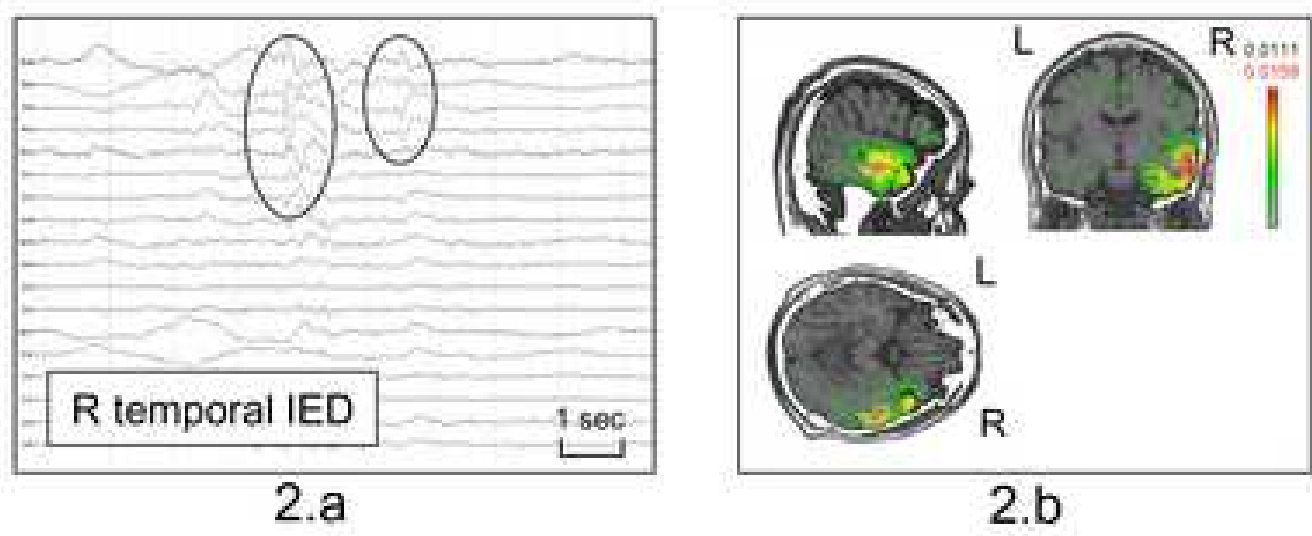

Figure 2

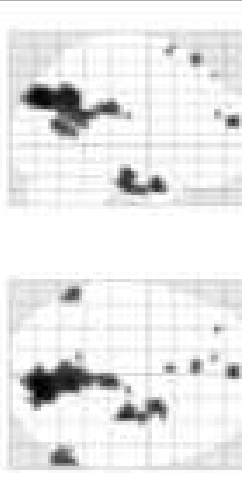

cESI model:

cESI+IED SPM $\{F\}$
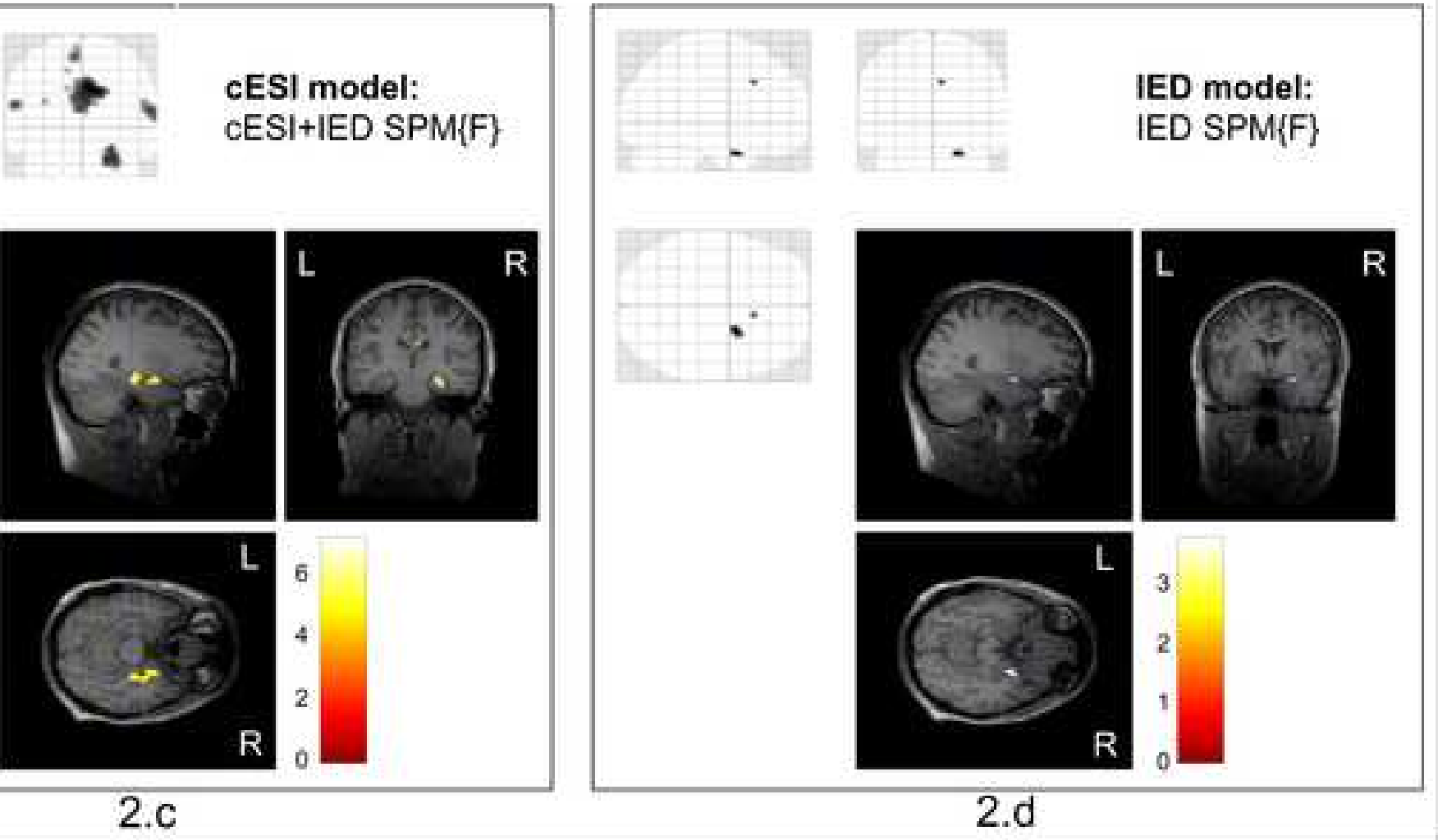

2.c

L

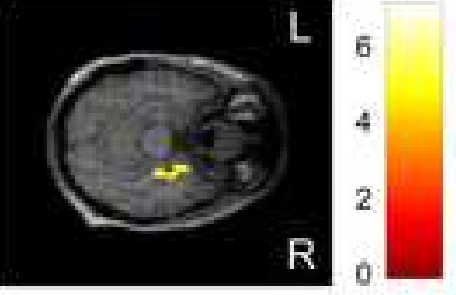




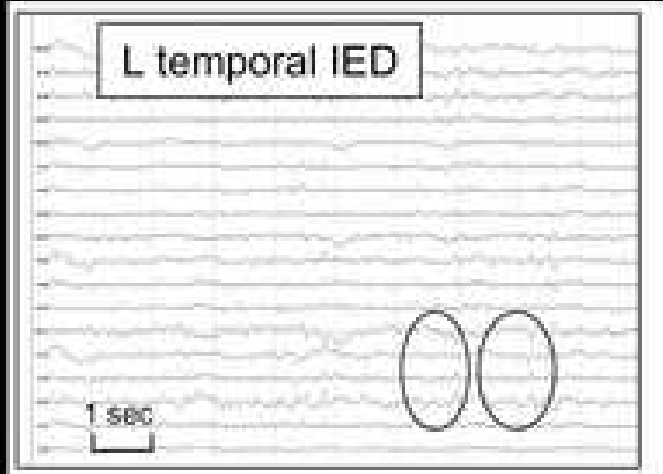

3.a

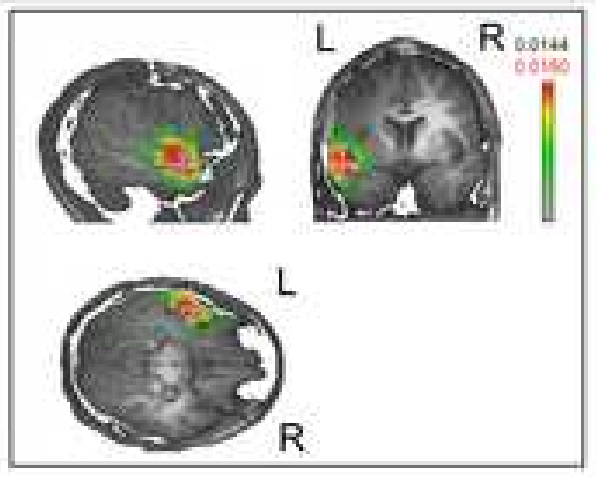

3.b

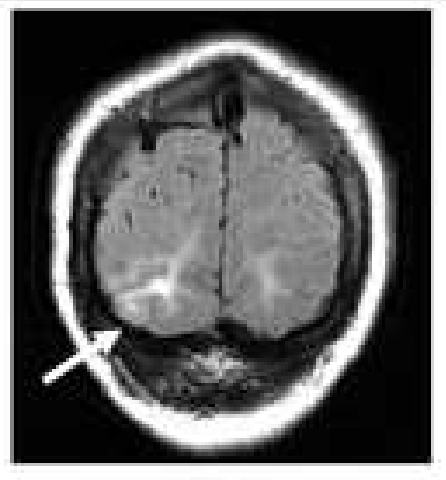

3.c

Figure 3

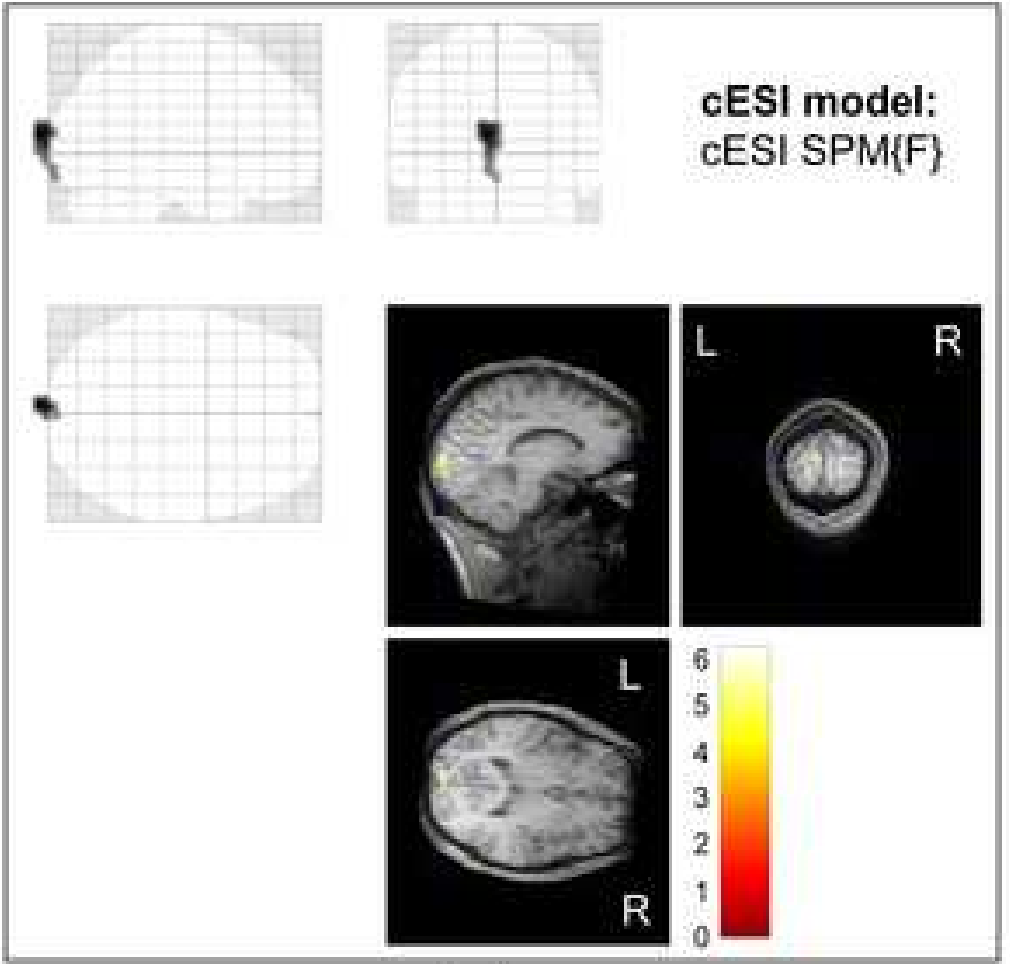

3.d

IED model:

IED SPM-F: not significant

3.e 


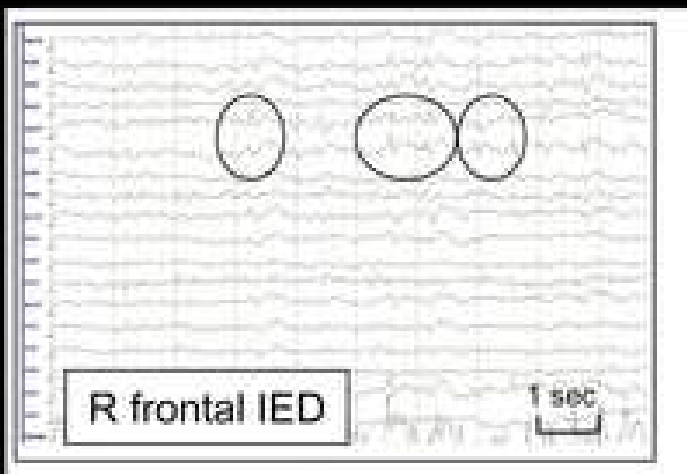

4.a

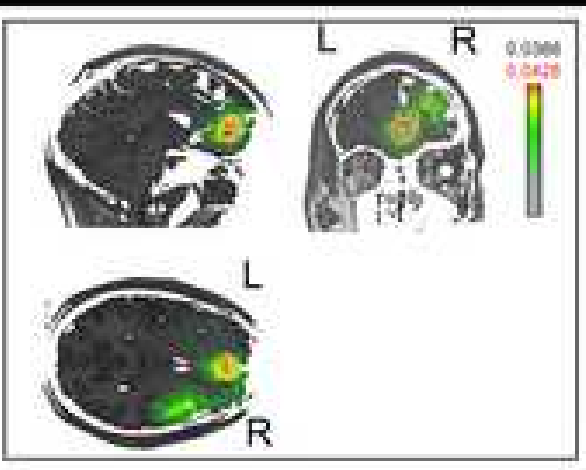

4.b

Figure 4

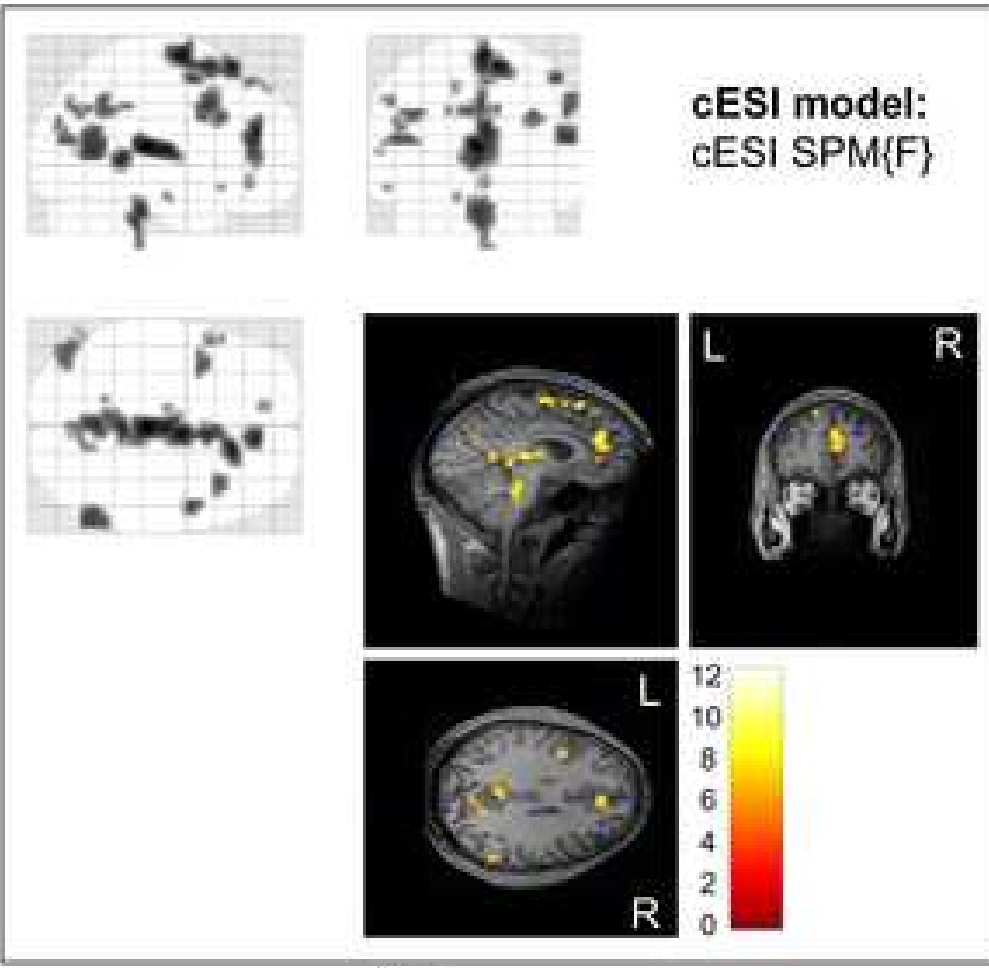

4.c

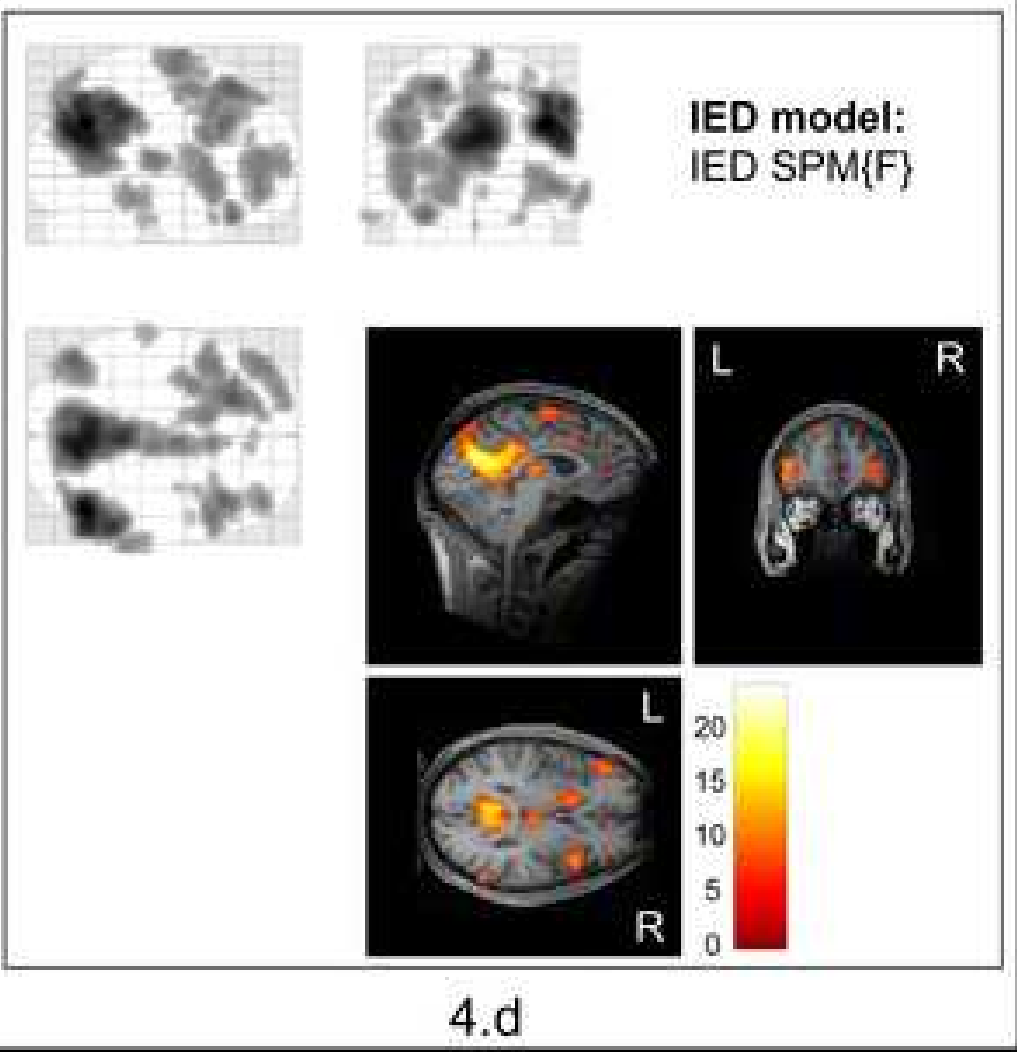

IED SPM $\{F\}$

R 


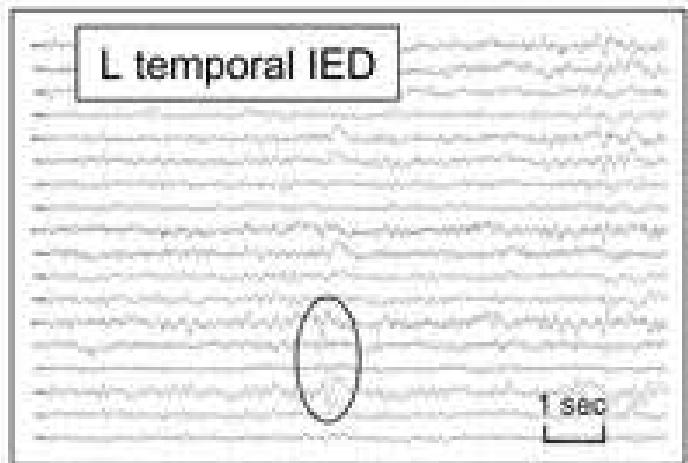

$5 . a$

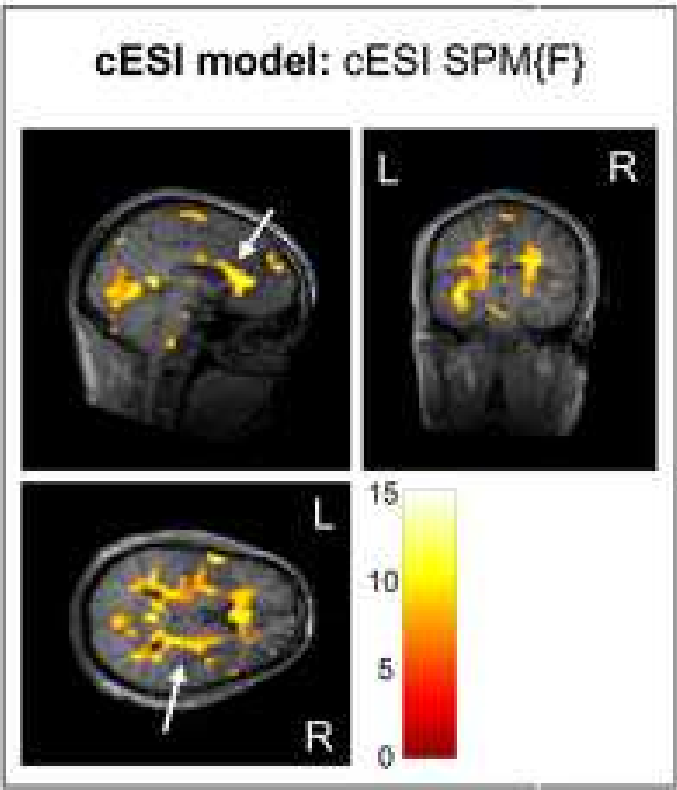

5.c
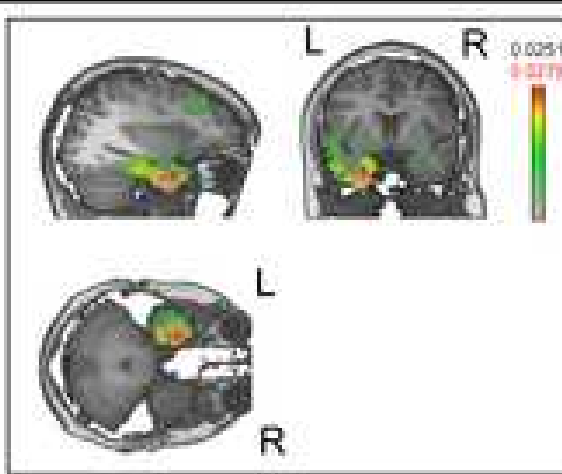

5.b

Figure 5

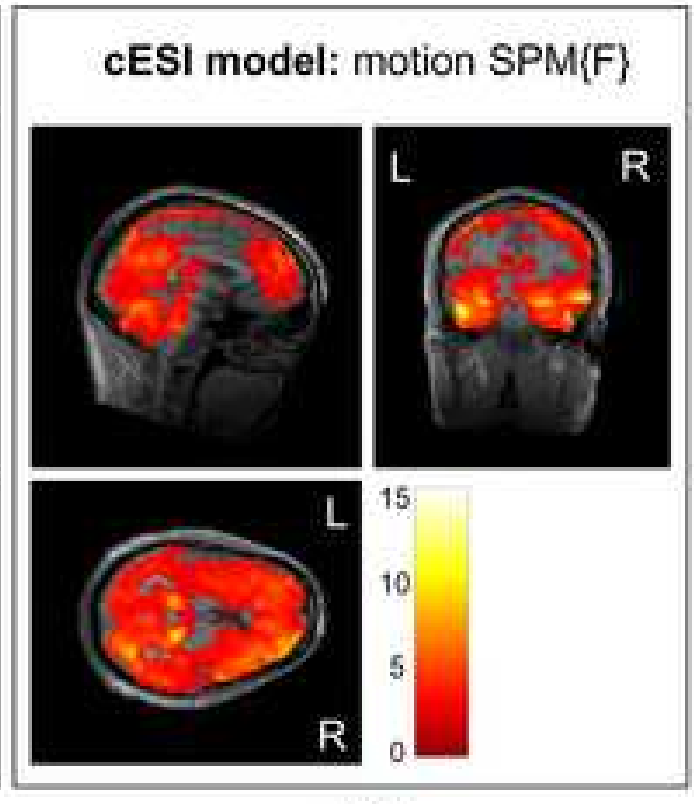

cESI model: cardiac SPM $\{F\}$
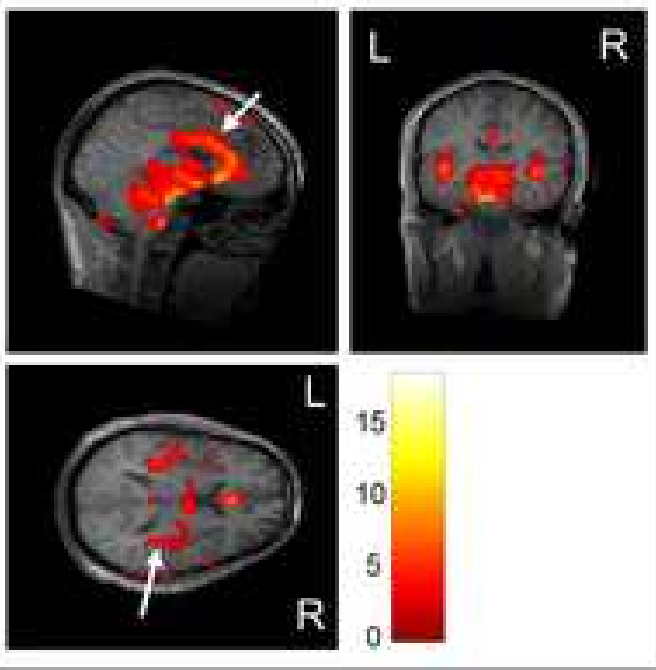

5.e

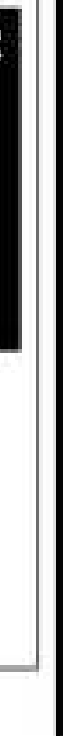




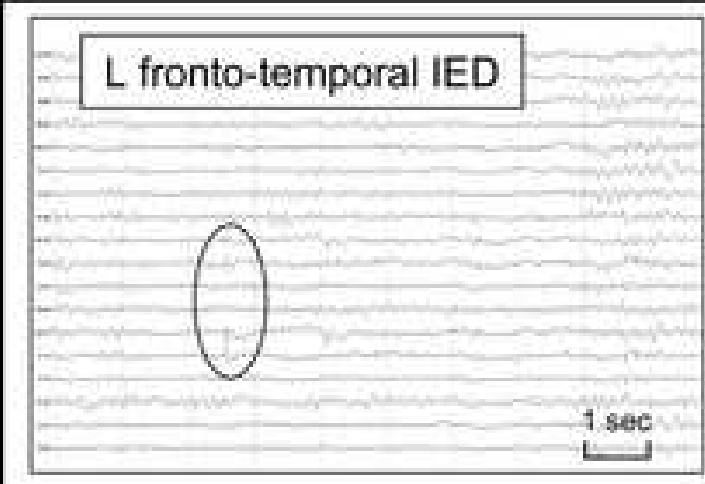

6.a

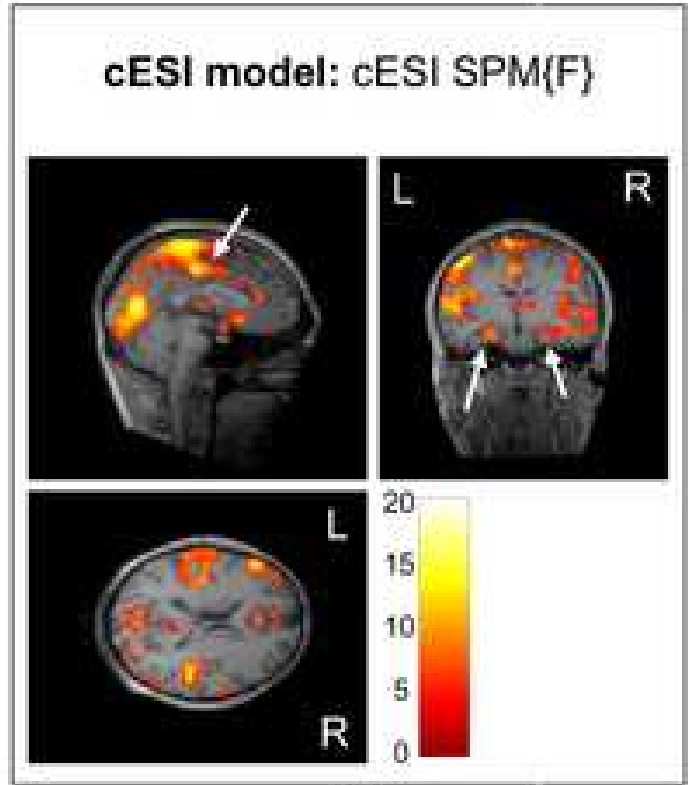

6.c

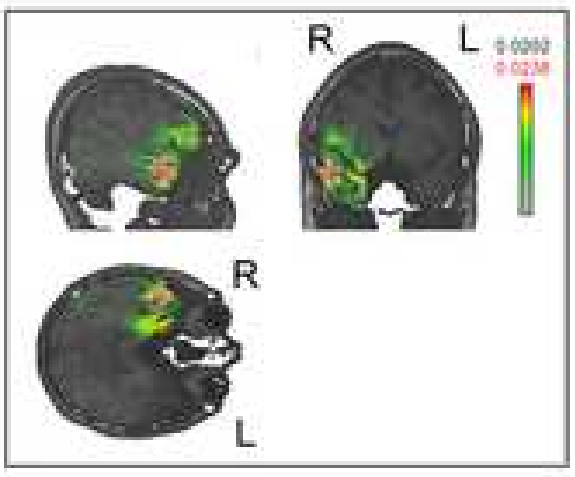

6.b

Figure 6

\section{cESI model: motion SPM $\{F\}$}
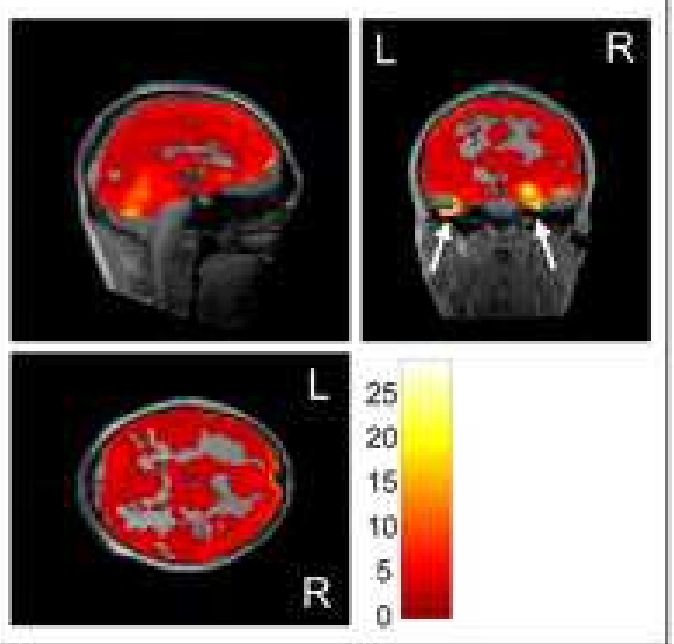

6.d
cESI model: cardiac SPM $\{F\}$
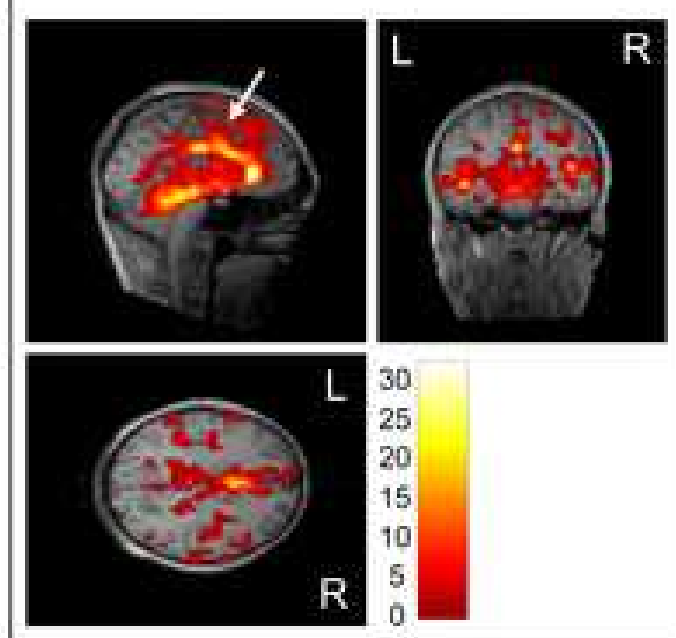

6.e 